茼

SSCL-Preprint-554

February 1994

Distribution Category: 400

G. López

Dissipative Model for a Two Component Gas System with Friction
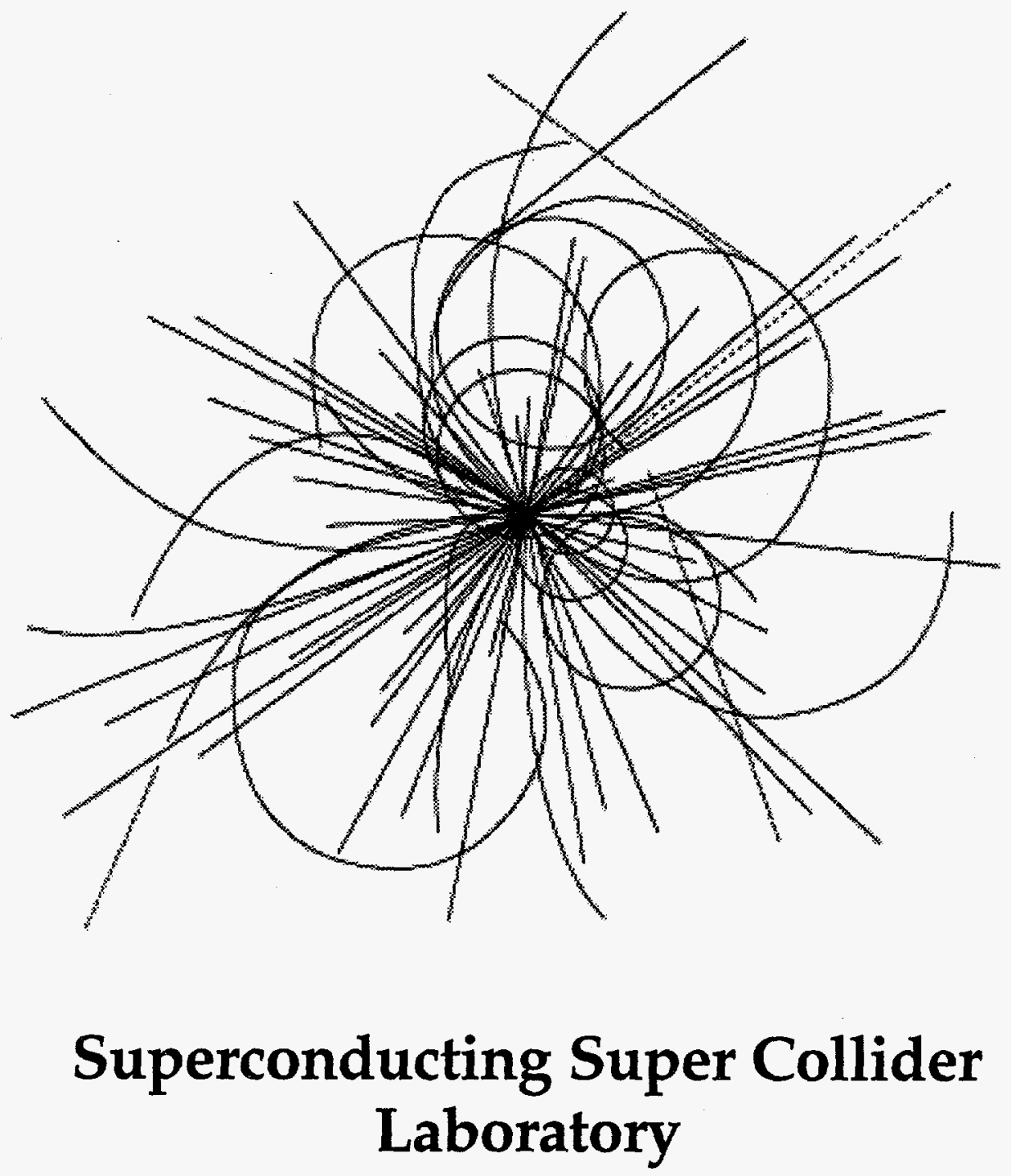

APPROVED FOR RELEASE OR PUBLICAIHON - O.R. PATEIY GROUP BY. $\triangle$ DATE. 40.060 


\section{Disclaimer Notice}

This report was prepared as an account of work sponsored by an agency of the United States Government. Neither the United States Government or any agency thereof, nor any of their employees, makes any warranty, express or implied, or assumes any legal liablity or responsibility for the accuracy, completeness, or usefulness of any iniormation, apparatus, product, or process disclosed, or represents that its use would not infringe privately owned rights. Reference herein to any specific commercial product, process, or service by trade name, trademark, manufacturer, or otherwise, does not necessarily constitute or imply its endorsement, recommendation, or favoring by the United States Govemment or any agency thereof. The views and opinions of authors expressed herein do not necessarily state or reflect those of the United States Govemment or any agency thereot.

Superconducting Super Collider Laboratory is an equal opportunity employer. 


\section{DISCLAIMER}

Portions of this document may be illegible in electronic image products. Images are produced from the best available original document. 


\title{
Dissipative Model for a Two Component Gas System with Friction*
}

\author{
G. López \\ Superconducting Super Collider Laboratory ${ }^{\dagger}$ \\ 2550 Beckleymeade Avenue \\ Dallas, Texas 75237 \\ and \\ M. Murgia $\ddagger$ and M. Sosa \\ Instituto de Física, Universidad de Guanajuato \\ Apartado Postal E-143, 37000 León, Gto., México
}

February 1994

* To be submitted to PHYSICA $A$.

$\dagger$ Operated by the Universities Research Association, Inc., for the U.S. Department of Energy under Contract No. DE-AC35-89ER40486.

$\ddagger$ Present address: Instituto Tecnológico de Monterrey, Campus Irapuato, Irapuato Gto., México.

$\S$ Present address: Fermilab, P.O. Box 500, MS-221, Batavia, Illinois 60510, U.S.A. 


\title{
Dissipative model for a two component gas system with friction
}

\author{
G. López \\ Superconducting Super Collider Laboratory ${ }^{\dagger}$ \\ 2550 Beckleymeade Avenue \\ Dallas, Texas 75237 \\ and \\ M. Murgia $a^{\ddagger}$ and M. Sosa $\AA^{\S}$ \\ Instituto de Física, Universidad de Guanajuato \\ Apartado Postal E-143, 37000 León, Gto., México
}

\begin{abstract}
For a mixture of small and large component of gases, the large component is treated as an ideal gas. A dissipative model is proposed for the small component, and the thermodynamic characteristics of the gas are derived through a statistical mechanical approach. The model requires for the small component to have a big dimension and its number of particles to be smaller than the large one. Using the associated partition function, the internal energy and the equation of state are calculated. The internal energy does not suffer any deviation from that of the two component ideal gas, but the equation of state deviates from that of the ideal gas for large values of the parameter which characterizes the dissipative model.
\end{abstract}

† Operated by the Universities Research Association, Inc., for the U.S. Department of Energy under Contract No. DE-AC35-89ER40486.

$\ddagger$ Present address: Instituto Tecnológico de Monterrey, Campus Irapuato, Irapuato Gto., México.

$\S$ Present address: Fermilab, P.O. Box 500, MS-221, Batavia, Illinois 60510, U.S.A. 


\section{Introduction}

Dealing with statistical mechanical problems [1] of systems in equilibrium, internal dissipative models for internal particle collision systems are not used because it is thought that dissipation must be associated with systems out of equilibrium. Therefore, a time depedent approach, Boltzman [2], Vlasov [3], or Fokker-Planck [4], is used to find the velocity distribution of the particles and the thermodynamic characteristics of the system.

Recently, it has been demonstrated in a series of works, López [5-10], that the Hamiltonian associated to a dissipative system does not necessarily have to be time dependent. Therefore, for an internal dissipative system having an associated time independent Hamiltonian which is a constant of motion of the system, it is possible to use the time independent approach and the usual statistical mechanic to find the thermodynamic characteristics of the system. This is the approach that will be used below in the study of a two-component gas system.

If a closed system is composed of two kinds of non-interacting particles, except for collisions and with very marked differences in number, dimensions, and masses, then it may be thought that the heavy-gross small number of particles are moving through a very light medium formed by the other particles. This medium produces some type of frictional force as a consequence of the average collisions with the heavy-gross particles. Thus, making a model for this motion, a time independent Hamiltonian is given, and the canonical ensemble is used to find the thermodynamic characteristics of this system. This type of system may not be just a mathematical curiosity since for example, high energy particle multiwire proportional chamber detectors containing Argon $(A r)$ with small quantities of Freon $\left(C F_{3} B_{r}\right)$ are normally used in laboratories [11,12]. In addition, the atmospheres of some of our planets [13] are highly rarified. A dissipative model may be useful in these or other cases where velocity depending frictional forces (dissipation) may appear. 


\section{Partition function, internal energy, and equation of state}

Assume a mixture of two types of particles, $N_{1}$ small particles of mass $m_{1}$ and $N_{2}$ biggross particles of mass $m_{2}$, contained in a volume of dimension $V$. The small particles move freely except for collisions with the walls of the container. The big particles move through the dissipative medium formed by the small particles with no other forces acting on them. The number of big particles will be assumed smaller than the number of small particles so that the approach has some sense. Assume, in addition, that the dissipative force in any particular direction, $i=x, y, z$, is proportional to the square of the velocity component in that direction. Therefore, the Newton's equations of motions for these particles can be written as

$$
m_{1} \frac{d^{2} q_{1 i j}}{d t^{2}}=0 \quad j=1, \ldots, N_{1}
$$

and

$$
m_{2} \frac{d^{2} q_{2 i k}}{d t^{2}}=-\alpha\left(\frac{d q_{2 i k}}{d t}\right)^{2} \quad k=1, \ldots, N_{2}
$$

where $q_{1 i j}, q_{2 i k}, d q_{1 i j} / d t$, and $d q_{2 i k} / d t$ are the generalized coordinates and the velocity components of the small (1) and big (2) particles. $\alpha$ is the parameter which characterizes the dissipative force. The Hamiltonian for the small particles is clearly given by

$$
H_{1}(q, p)=\sum_{j=1}^{N_{1}} \sum_{i=1}^{3} \frac{p_{1 i j}^{2}}{2 m_{1}} .
$$

As it can be shown [5], the constant of motion for the big particles can be expressed as

$$
K_{2}(q, v)=\sum_{k=1}^{N_{2}} \sum_{i=1}^{3} \frac{1}{2} m_{2} v_{2 i k}^{2} \exp \left(2 \alpha q_{2 i k} / m_{2}\right),
$$

where $v_{2 i k}$ represents the ith-component of the velocity of the kth-big particle. Using the formulation given in reference [6] for the classical mechanics, the Lagrangian expression for a single particle and component in terms of the constant of motion is given by

$$
L(q, v)=v \int^{v} \frac{K(q, \xi)}{\xi^{2}} d \xi
$$


Therefore, using (4) and the generalization of Eq. (5) to a multidimensional space [9], the following expression is gotten for the Lagrangian of the system (2),

$$
L_{2}(q, v)=\sum_{k=1}^{N_{2}} \sum_{i=1}^{3} \frac{1}{2} m_{2} v_{2 i k}^{2} \exp \left(2 \alpha q_{2 i k} / m_{2}\right) .
$$

As a result, the generalized momentum and Hamiltonian follow,

$$
p_{2 i k}=m_{2} v_{2 i k} \exp \left(2 \alpha q_{2 i k} / m_{2}\right)
$$

and

$$
H_{2}(q, p)=\sum_{k=1}^{N_{2}} \sum_{i=1}^{3} \frac{p_{2 i k}^{2}}{2 m_{2}} \exp \left(-2 \alpha q_{2 i k} / m_{2}\right) .
$$

The Hamiltonian of Eq. (3) corresponds to a system of $N_{1}$ particles moving freely in a container of volume $V$, meanwhile Eq. (8) is the Hamiltonian of a system of $N_{2}$ particles moving in a dissipative medium. Knowing the Hamiltonian, the canonical partition function can be calculated from the known relation [1]

$$
Z=\frac{1}{N_{1} ! N_{2} ! h^{3 N}} \int \exp (-\beta H) d q d p
$$

where $H$ is the total Hamiltonian, $H=H_{1}+H_{2} . \quad h$ is the Planck's constant. $N$ is the total number of particles, $N=N_{1}+N_{2}$, and $\beta$ is given by

$$
\beta=1 / K T,
$$

where $K$ is the Boltzmann's constant, and $T$ is the temperature. The differentials $d q$ and $d p$ are defined as

$$
d q=\prod_{j=1}^{N_{1}} \prod_{i=1}^{3} d q_{1 i j} \prod_{k=1}^{N_{2}} \prod_{i=1}^{3} d q_{2 i k}
$$

and

$$
d p=\prod_{j=1}^{N_{1}} \prod_{i=1}^{3} d p_{1 i j} \prod_{k=1}^{N_{2}} \prod_{i=1}^{3} d p_{2 i k}
$$


The spatial integration in Eq. (9a) is carried out over the volume $V$. Substituting Eq. (3) and Eq. (8) in Eq. (9a), and taking into consideration that many integrations are repeated in form, Eq. (9a) can be written as

$$
\begin{aligned}
Z= & \frac{1}{N_{1} ! N_{2} ! h^{3 N}} \prod_{j=1}^{N_{1}} \prod_{i=1}^{3} \int \exp \left(-\beta p_{1 i j}^{2} / 2 m_{1}\right) d q_{1 i j} d p_{1 i j} \\
& \times \prod_{k=1}^{N_{2}} \prod_{i=1}^{3} \int \exp \left(-\beta p_{2 i k}^{2} / 2 m_{2}\right) \exp \left(-2 \alpha q_{2 i k} / m_{2}\right) d q_{2 i k} d p_{2 i k} .
\end{aligned}
$$

Performing the above integrations, the following expression for the partition function is gotten

$$
Z=\frac{V^{N}}{N_{1} ! N_{2} !}\left(\frac{2 \pi m_{1}}{\beta h^{2}}\right)^{N_{1} / 2}\left(\frac{2 \pi m_{2}}{\beta h^{2}}\right)^{N_{2} / 2}\left(\frac{m_{2}}{\alpha V^{1 / 3}}\right)^{3 N_{2}}\left[\exp \left(\alpha V^{1 / 3} / m_{2}\right)-1\right]^{3 N_{2}}
$$

The internal energy and the equation of state can be easily calculated from the relations

$$
U=-\left(\frac{\partial \log Z}{\partial \beta}\right)
$$

and

$$
P=\frac{1}{\beta}\left(\frac{\partial \log Z}{\partial V}\right)
$$

where $P$ represents the pressure of the thermodynamic system. Using the partition function (11) in Eq. (12) and Eq. (13), and making some rearrangements, the internal energy and the equation of state have the following expressions

$$
U=\frac{1}{2} N K T
$$

and

$$
P=\frac{N K T}{V}+N_{2} K T \psi\left(V, \alpha / m_{2}\right)
$$

where the function $\psi$ has been defined as

$$
\psi\left(V, \alpha / m_{2}\right)=\frac{1}{V}+\frac{\alpha V^{-2 / 3}}{m_{2}\left[1-\exp \left(-\alpha V^{1 / 3} / m_{2}\right)\right]} .
$$

As can be seen from Eq. (14) and Eq. (15a), there is no modification in the internal energy of the sytem with respect to the ideal gas. However, the equation of state suffers 
a modification due to the dissipation in the system. This modification, however, is only noticeable for very unrealistically high values of $\alpha$. On the other hand, it is clearly seen from Eq. (15b) that if the parameter $\alpha$ goes to zero, the following limit is gotten

$$
\lim _{\alpha \rightarrow 0} \psi\left(V, \alpha / m_{2}\right)=0
$$

That is, within this limit Eqs (2), (4), (6), (7), (8), (9), (12), and (16a) take their usual form for an ideal gas. For dissipation effects to become important in this model, the value of the volume and friction coefficients must be such that

$$
\frac{\alpha V^{1 / 3}}{m_{2}}>1
$$

In this case, the exponential term appearing in Eq. (15b) can be neglected, and the equation of state has the form

$$
P \approx \frac{N_{1} K T}{V}+\frac{\alpha N_{2} K T}{m_{2} V^{2 / 3}}
$$

which can be written in terms of the component densities, $n_{1}=N_{1} / V$ and $n_{2}=N_{2} / V$, as

$$
\frac{P}{n_{1} K T}=1+\alpha\left(\frac{n_{1}}{n_{2}}\right) \frac{V^{1 / 3}}{m_{2}} .
$$

From Eqs. (15a) and (19), it is clear that the system is not invariant under spatial divisions since the right hand of these equations depends on the density and the volume of the system, i.e., even if the density ratio $n_{2} / n_{1}$ and the densities themselves are kept constant, the pressure of the system would depend on the volume of the system.

\subsection{Conclusion}

A model for an internal dissipative system of two-component gas has been studied. This model has meaning if it is possible to assume that one of the components is able to produce a classical frictional force on the other component, as an average effect. The justification for the canonical ensemble to be used comes from the Lioville's theorem and using the fact the Hamiltonian is independent of time and a constant of motion of the system. The 
internal energy of the system (which is better called generalized internal energy since it is used in association to a dissipative system) has the same expression like that of an ideal gas, but the resulting equation of state differs from that of an ideal gas for high dissipation (if Eq. (17) is satisfied). The correct limit (ideal gas) is obtained when the dissipation parameters go to zero. 


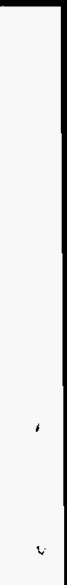




\section{REFERENCES}

[1] K. Huang, Statistical Mechanics, John Wiley \& Sons Inc., New York-London, (1963), part B.

[2] C. Kittel, Elementary Statistical Physics, John Wiley \& Sons Inc., New York-London, (1958), part 2.

[3] F. Reif, Fundamentals of Statistical and Thermal Physics, McGraw-Hill, (1965), chapter 13.

[4] J. J. Durderstand and W. R. Martin,Transport Theory, John Wiley \& Sons Inc., New York-London, (1979), chapter 3.

[5] G. López, Perturbation Approach and the Constant of Motion for One-Dimensional Dynamical System, SSCL-PP-14, November (1991).

[6] G. López, Hamiltonian and Lagrangian for One Dimensional Autonomous Systems and Dissipative Systems, SSCL-472, (1991).

[7] G. López and J. I. Hernández, Ann. of Phys., 193 (1989)1.

[8] G. López, Constant of Motion for One-Dimensional and nth-Order Autonomous System, and Its Relation to the Lagrangian and Hamiltonian, SSCL-543, (1993).

[9] G. López, Constant of Motion, Hamiltonian and Lagrangian for Autonomous System Defined in a Hyperbolic Flat Space, SSCL-150, (1993).

[10] G. López, Constant of Motion and Dynamic Equations for One-Dimensional Autonomous System and Radiation Damping, SSCL-308, (1990).

[11] F. Sauli, CERN Report, 77-09, (1977).

[12] F. Ferbel, Experimental Techniques in High Energy Nuclear and Particle Physics, World Scientific, Second Edition, (1991), chapter 2.

[13] T. Encrenaz, J. Bibring, and M. Blanc, The Solar System, Springer-Verlag, (1989). 